\title{
A new biotransformation mode of zearalenone identified in Bacillus subtilis Y816 revealing a novel conjugate $\mathrm{ZEN}$ conjugate
}

ShiBin Yang ${ }^{1,3, \#}$ HongChen Zheng ${ }^{1,2,3,4, \#, *}$ JianYong $\mathrm{Xu}^{2,3}$ XingYa Zhao ${ }^{3,4}$ WenJu Shu ${ }^{3,4}$ Xiangming Li $^{5}$ Hui Song ${ }^{1,2,3,4, *}$ YanHe $\mathrm{Ma}^{2,3, *}$

${ }^{1}$ University of Chinese Academy of Sciences, Beijing 100049, China

${ }^{2}$ National Technology Innovation Center of Synthetic Biology, Tianjin Institute of Industrial Biotechnology, Chinese Academy of Sciences, Tianjin 300308, China

${ }^{3}$ Industrial Enzymes National Engineering Laboratory, Tianjin Institute of Industrial Biotechnology, Chinese Academy of Sciences, Tianjin 300308, China

${ }^{4}$ Tianjin Key Laboratory for Industrial Biological Systems and Bioprocessing Engineering, Tianjin Institute of Industrial Biotechnology, Chinese Academy of Sciences, Tianjin 300308, China

${ }^{5}$ Preventive Medicine Department, Medical College, Yangzhou University, Yangzhou, Jiangsu 225001, China

\#ShiBin Yang and Hongchen Zheng contributed equally to this work and are listed as co-first authors.

${ }^{*}$ Corresponding authors. Address: No. 32 West 7th Avenue, Tianjin Airport Economic Area, Tianjin 300308, China. Tel: 086+022+84861934; Fax: 086+022+84861934. E-mail address: zheng_hc@tib.cas.cn (HongChen Zheng); song_h@tib.cas.cn (Hui Song); ma_yh@tib.cas.cn (YanHe Ma). 
Table S1. Characterastics of strain Y816 analyzed by the Biolog ${ }^{\circledR}$ automated microbe identification system

\begin{tabular}{cccc}
\hline Substrate & Result & Substrate & Result \\
\hline Pyruvic Acid Methyl Ester & - & D-Galacturonic Acid & + \\
Tween 40 & - & 2-Hydroxy Benzoic Acid & - \\
Tween 80 & + & 4-Hydroxy Benzoic Acid & + \\
a-Cyclodextrin & + & y-Hydroxybutyric Acid & + \\
Glycogen & - & Itaconic Acid & + \\
D-Cellobiose & + & a-Ketobutyric Acid & + \\
a-D-Lactose & - & D-Malic Acid & + \\
B-Methyl-D-Glucoside & + & L-Arginine & + \\
D-Xylose & + & L-Asparagine & + \\
I-Erythritol & + & L-Phenylalanine & - \\
D-Mannitol & + & L-Serine & - \\
N-Acetyl-D-Glucosamine & + & L-Threonine & - \\
D-Glucosaminic Acid & + & Glycyl-L-Glutamic Acid & - \\
Glucose-1-Phosphate & - & Phenylethyl-amine & + \\
D, L-a-Glycerol & + & Putrescine & - \\
D-Galactonic Acid y-Lactone & + & & \\
\hline
\end{tabular}

Note: "+" indicates a positive result; "-" indicates a negative result. The analysis result indicated that strain Y816 is Bacillus subtilis.

A

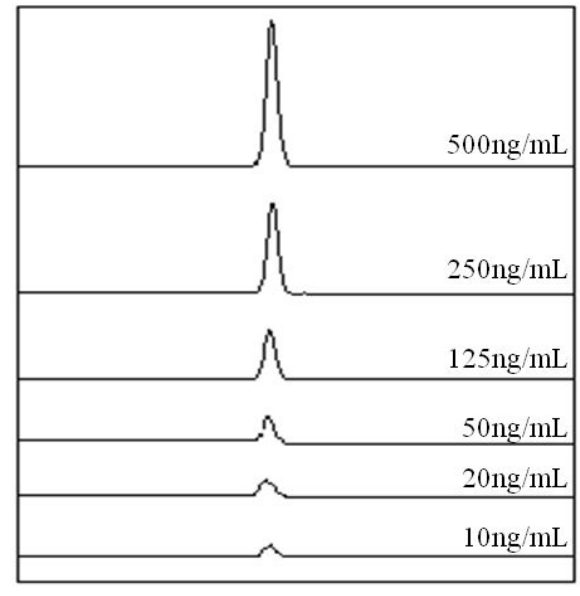

B

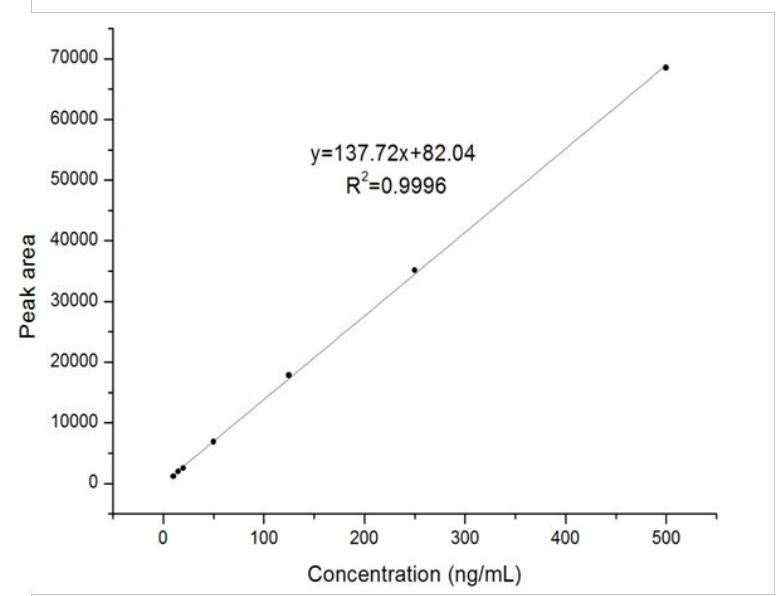

Figure S1. The standard curve of the concentration of ZEN by HPLC in this study. (A) The ZEN peak of different concentrations of ZEN detected by HPLC. (B) The linear equation between the concentrations of ZEN and the peak areas of ZEN 
detected by HPLC.
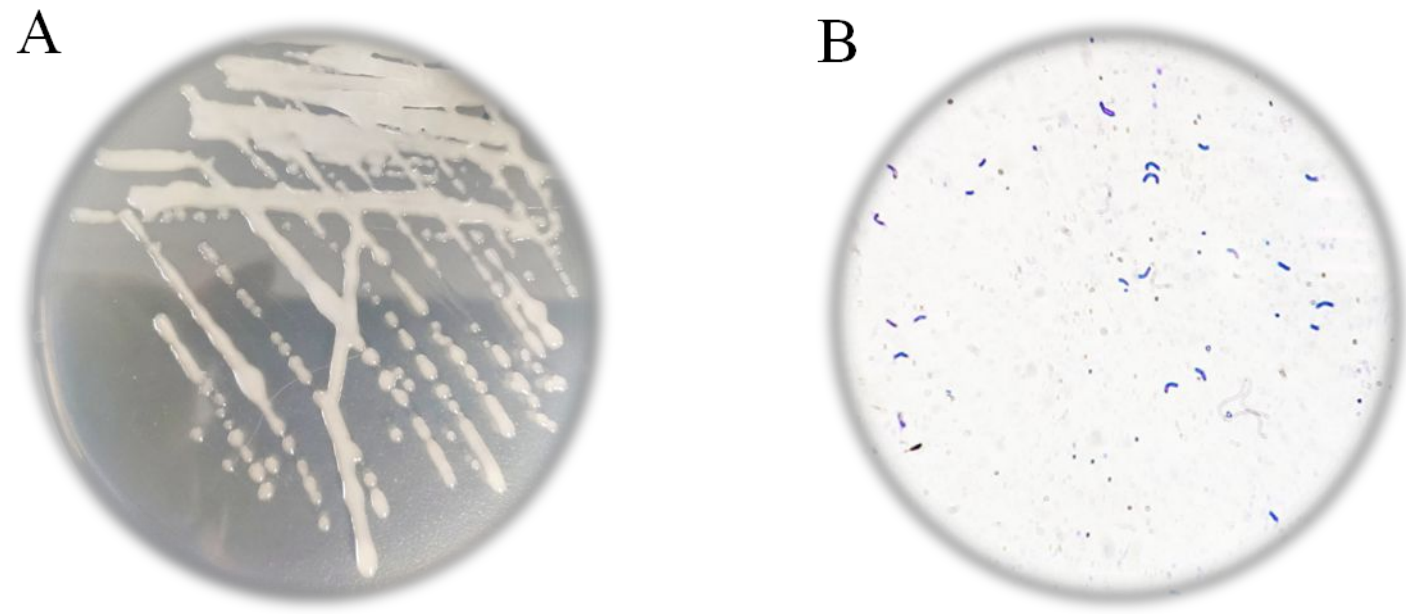

Figure S2. The morphological identification of strain Y816. (A) The colony morphology of strain Y816 in LB plate cultured at $37^{\circ} \mathrm{C}$ for $16 \mathrm{~h}$. The bacterial colony is beige, rounded and smooth in surface with neat edges. (B) The morphological observation of strain Y816 by gram's stain $(\times 1000)$. It belonged to gram-positive bacteria with a rod-shape, and could form spores in the cultivation process, suggesting a possibility of bacillus $s p$. strain.

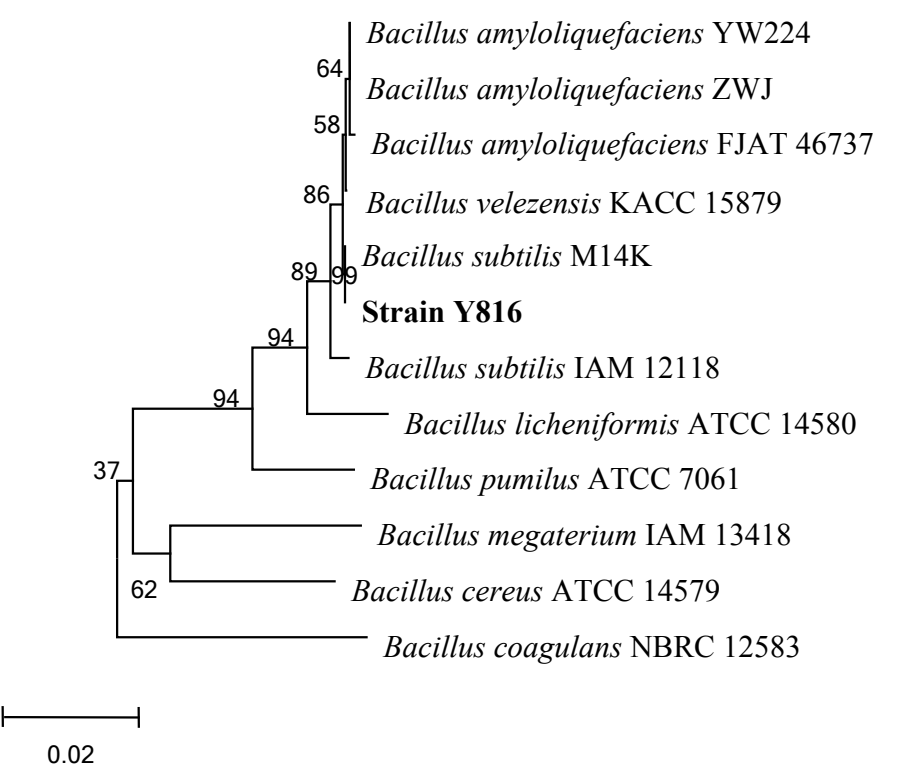

Figure S3. Phylogenetic tree of strain Y816 and its closest bacillus strains based on 16S rDNA gene sequences. Strain Y816 showed the highest similarity to Bacillus subtilis M14K with more than $99.9 \%$ identity. 

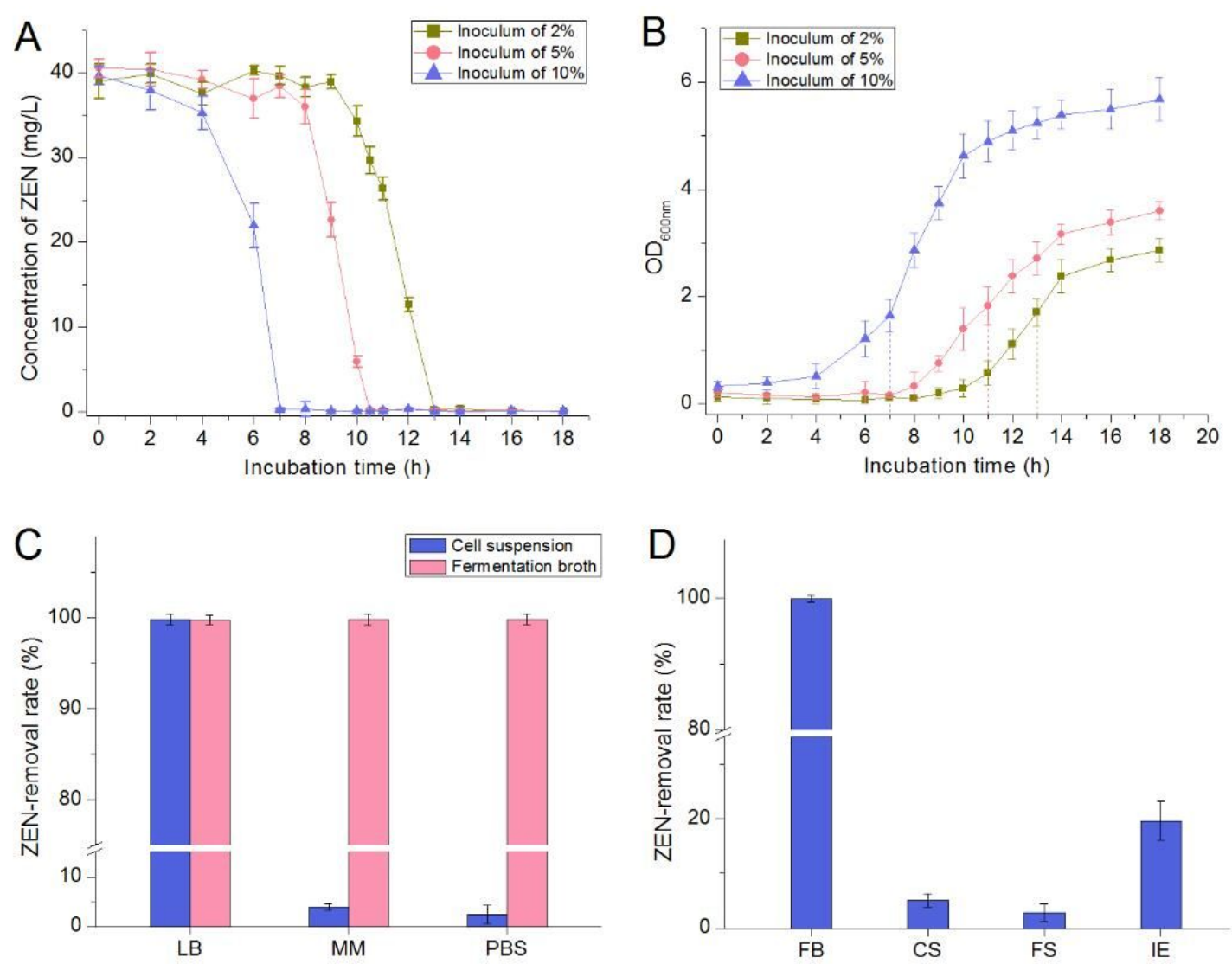

Figure S4. The ZEN biotransformation characteristics of strain Y816 in different conditions.

(A) Effects on ZEN biotransformation efficiency in different inoculated doses. (B) Different growth profiles of strain Y816 in different inoculated doses. The blue dotted line indicated the $\mathrm{OD}_{600 \mathrm{~nm}}$ value of strain $\mathrm{Y} 816$ at $7 \mathrm{~h}$ fermentation with inoculum of $10 \%$. The red dotted line indicated the $\mathrm{OD}_{600 \mathrm{~nm}}$ value of strain $\mathrm{Y} 816$ at $11 \mathrm{~h}$ fermentation with inoculum of $5 \%$. The green dotted line indicated the $\mathrm{OD}_{600 \mathrm{~nm}}$ value of strain Y816 at $13 \mathrm{~h}$ fermentation with inoculum of 2\%. (C) Effects on ZEN biotransformation efficiency in different media with the same inoculum concentration of fermentation broth and cell suspension of strain Y816. LB: LB medium, MM: minimal medium, PBS as a control: phosphate buffer (50 mM, pH7.0). (D) Different ZEN biotransformation rate by various elements of the fermentation broth of strain Y816. FB: fermentation broth, CS: cell suspension, FS: fermentation supernatants, IE: intracellular extract. All values are expressed in means $\pm \operatorname{SD}(n=3)$. 

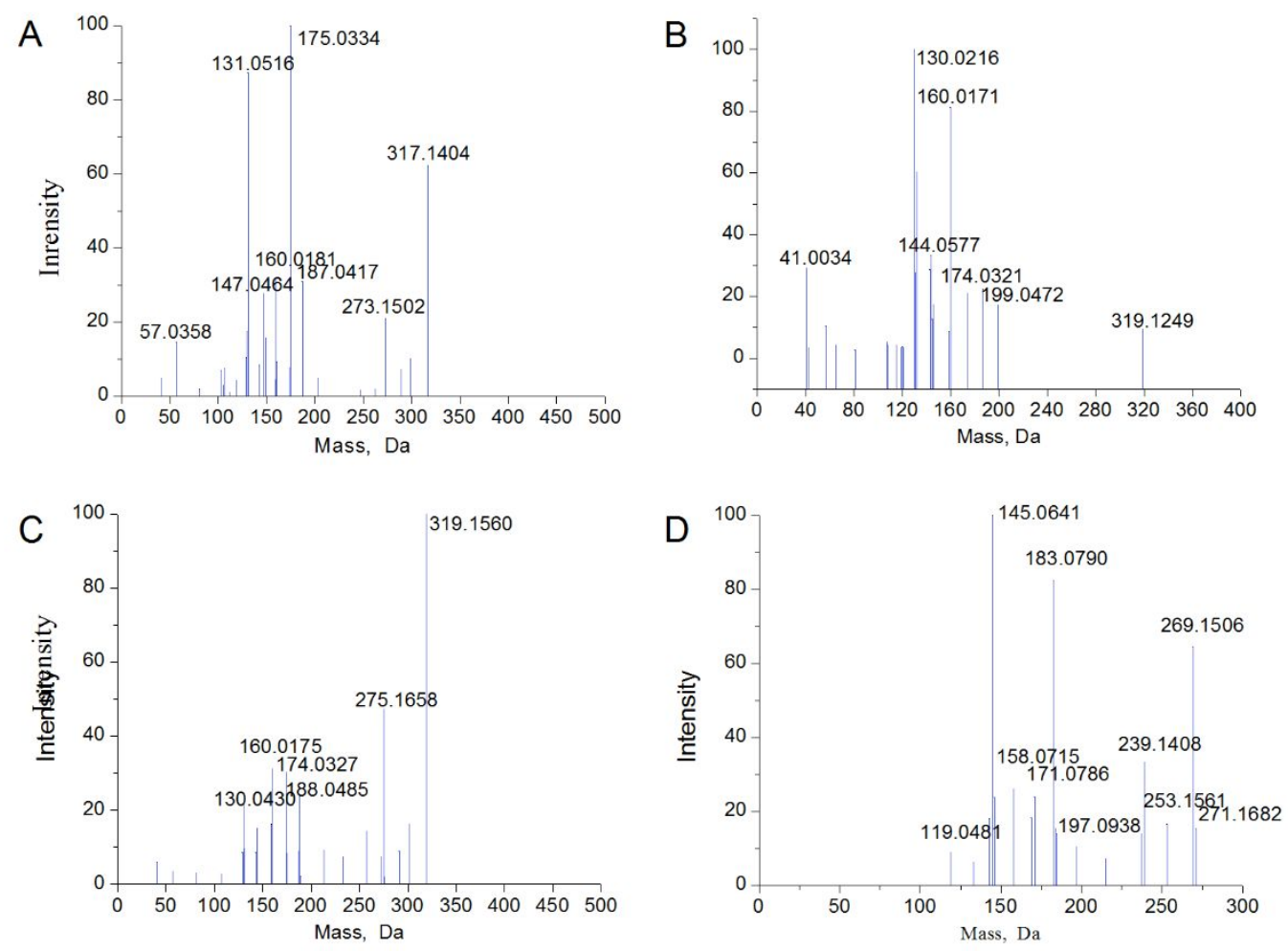

Figure S5. The secondary mass spectrometry of estrogenic substrates.

(A) Secondary mass spectrometry of ZEN. (B) Secondary mass spectrometry of $\alpha$-ZOL. (C) Secondary mass spectrometry of $\beta$-ZOL. (D) Secondary mass spectrometry of E2.
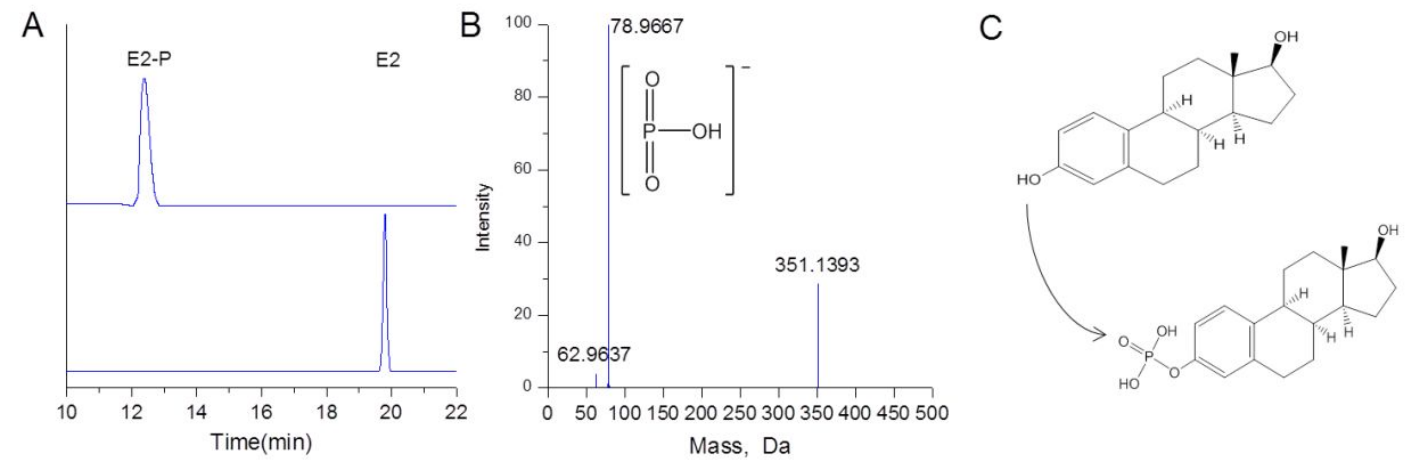

Figure S6. Identification of transformation product of E2 by HPLC-MS.

(A) HPLC analysis results of E2 before transformation and its transformation product (E2-P) after $12 \mathrm{~h}$ biotransformation by strain Y816. (B) Secondary mass spectrometry of E2-P. (C) The speculation of the transformation results according to the structure 
analysis of E2-P and E2 by MS and NMR.
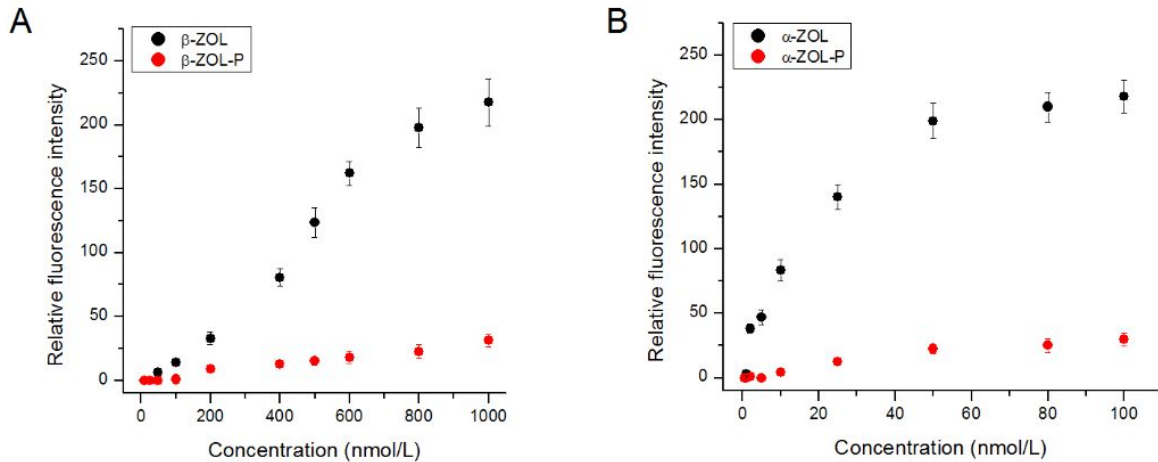

Figure S7. The comparison of estrogen activity between $\alpha / \beta-Z O L$ and $\alpha / \beta-Z O L-P$. (A) The relative estrogen activities of $\beta-Z O L$ and $\beta-Z O L-P$ at different concentrations of $0-100 \mathrm{nM}$. (B) The relative estrogen activities of $\alpha-Z O L$ and $\alpha-Z O L-P$ at different concentrations of $0-1000 \mathrm{nM}$. All values are expressed in means $\pm \operatorname{SD}(n=3)$.

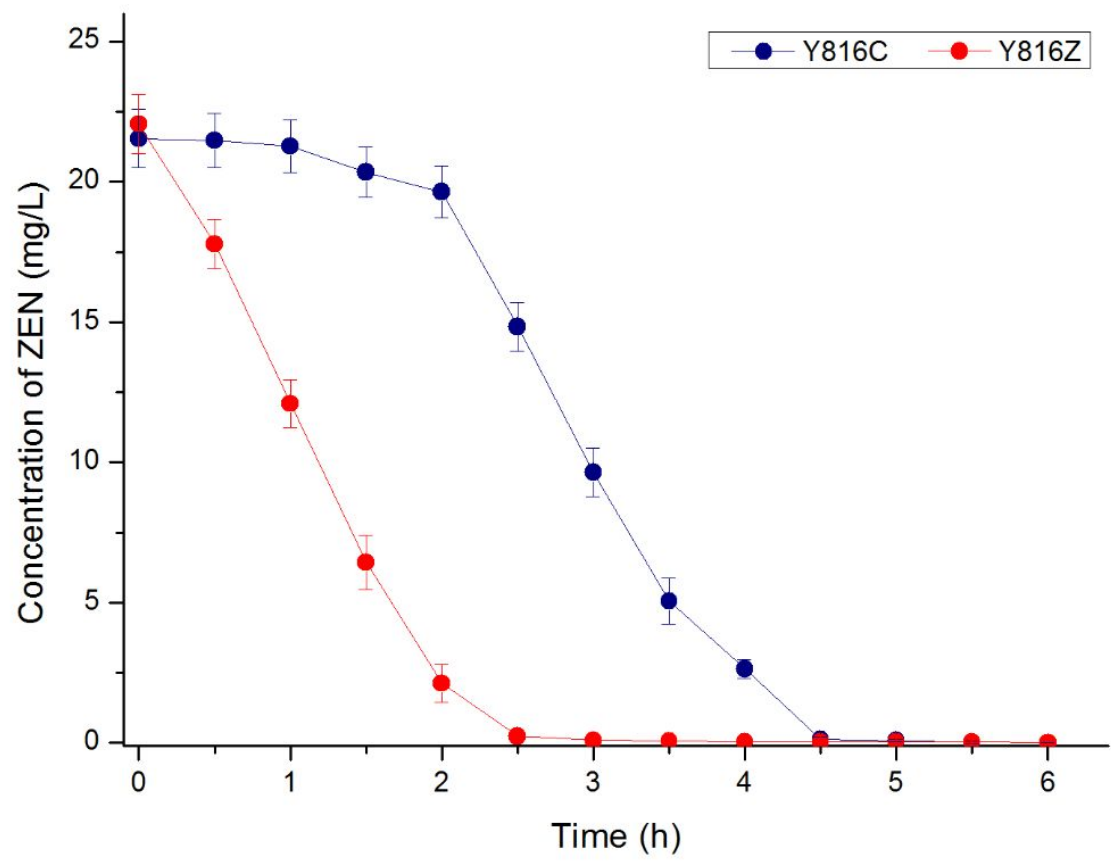

Figure S8. Biotransformation curve of ZEN in the fermentation of different inoculate strains.

Y816C: inoculating strain Y816 which has been cultured for $12 \mathrm{~h}$ without ZEN addition; Y816Z: inoculating strain Y816 which has been induced by $20 \mathrm{mg} / \mathrm{L}$ ZEN in $12 \mathrm{~h}$ fermentation. 


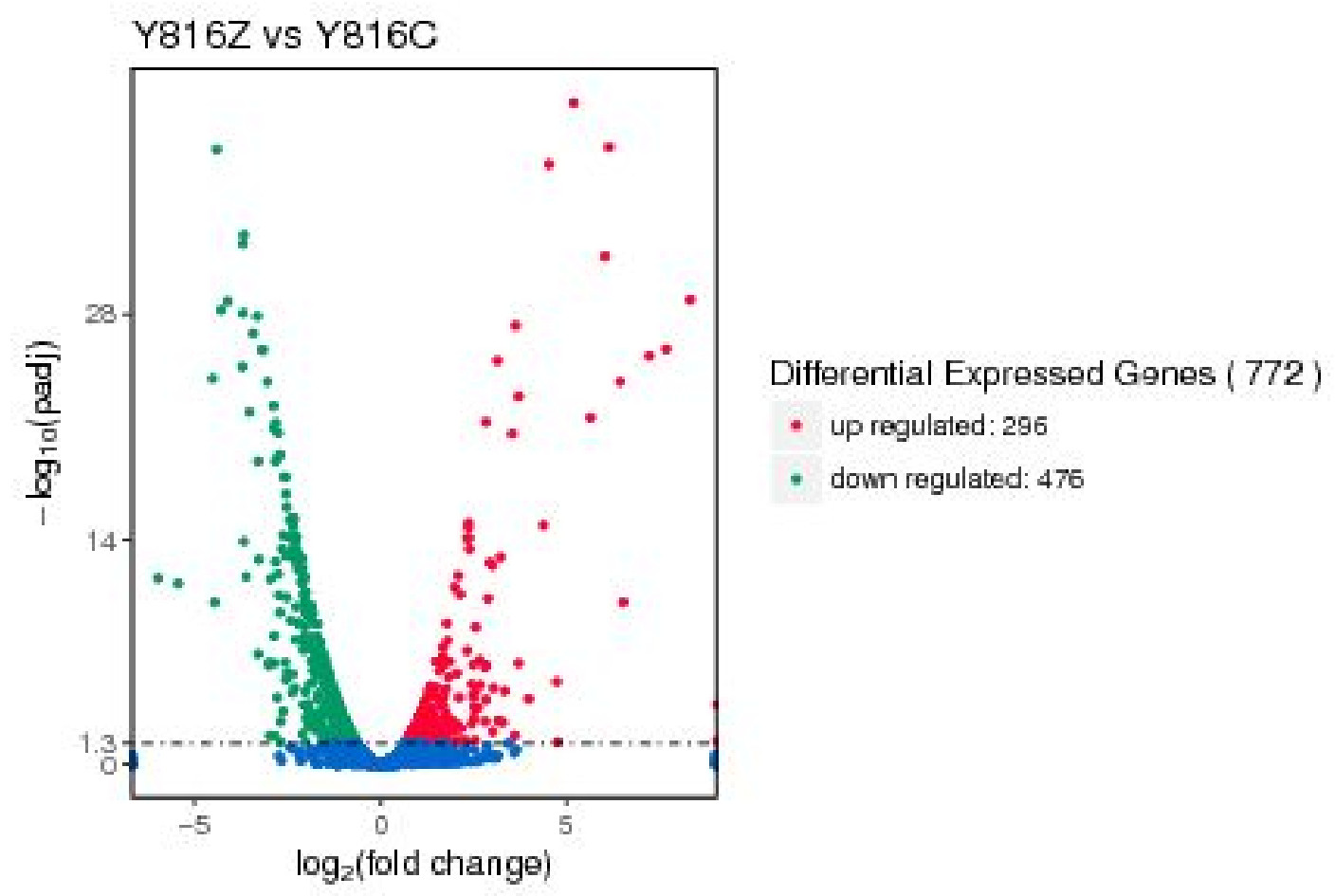

Figure S9. The volcano distributions of differential expressed genes basing on the compared transcriptome analysis.

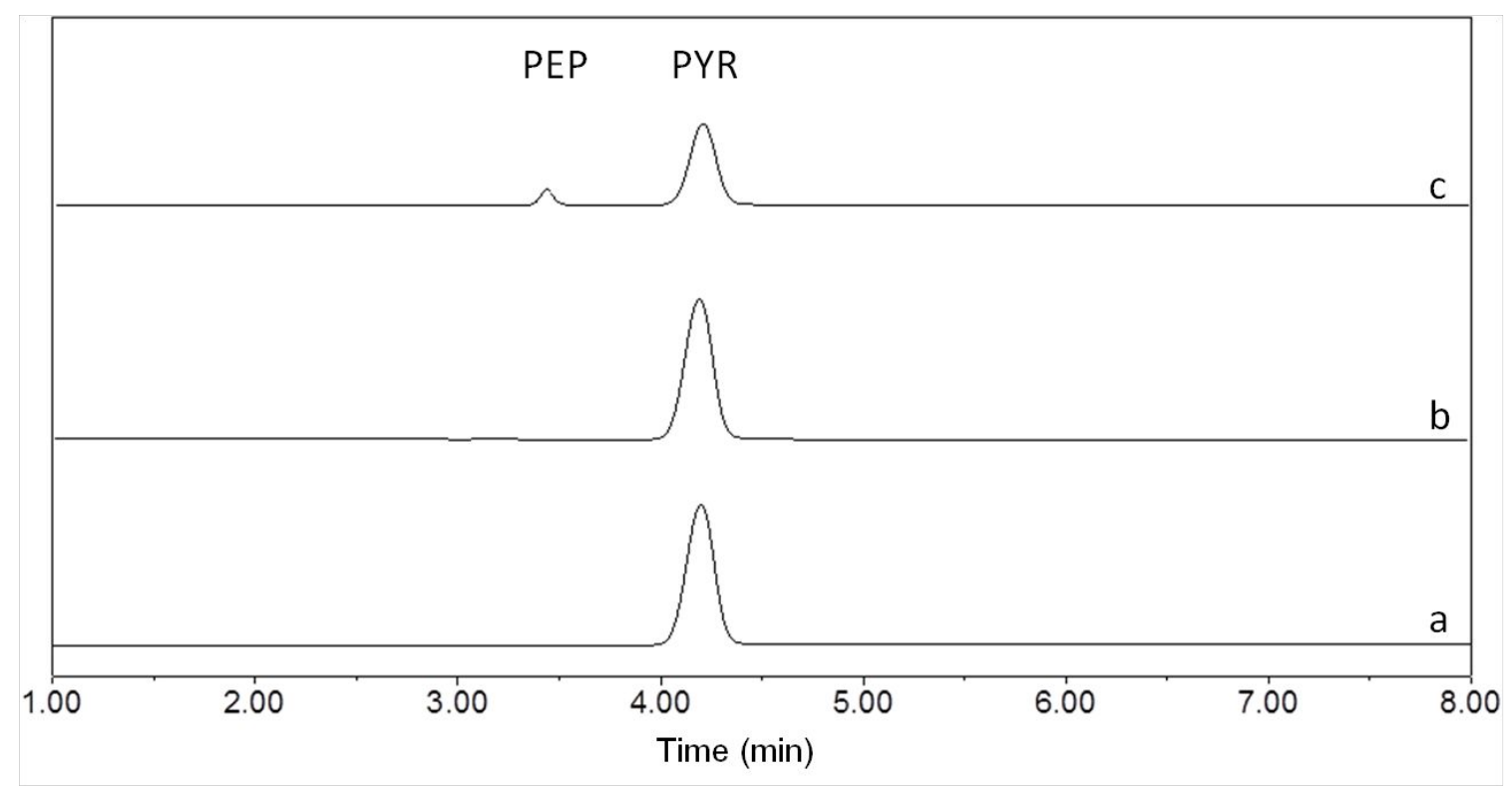

Figure S10. HPLC analysis of the enzymatic transform of pyruvate by ZPH. a: the initial determination before the transform reaction of $0.1 \mathrm{mM}$ pyruvate; $b$ : determine the sample after 5 min reaction in the reaction system of a; $c$ : the standard peaks of pyruvate and PEP. 


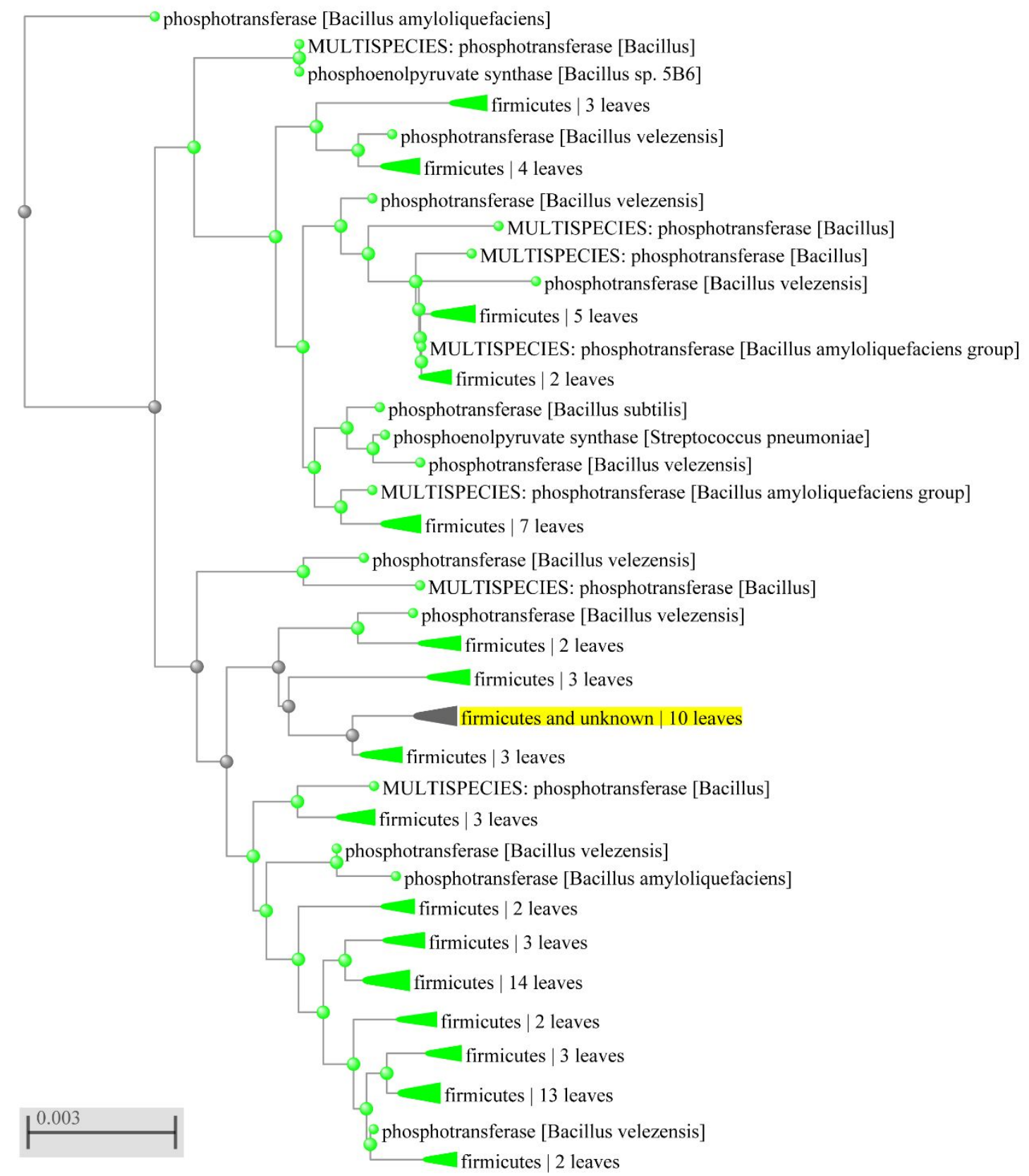

Figure S11. Distance tree of protein sequences of reported phosphotransferases with more than $98 \%$ sequence identity with $\mathrm{ZPH}$.

ZPH was in the yellow group. The distance tree was calculated by the method of Neighbor Joining. 


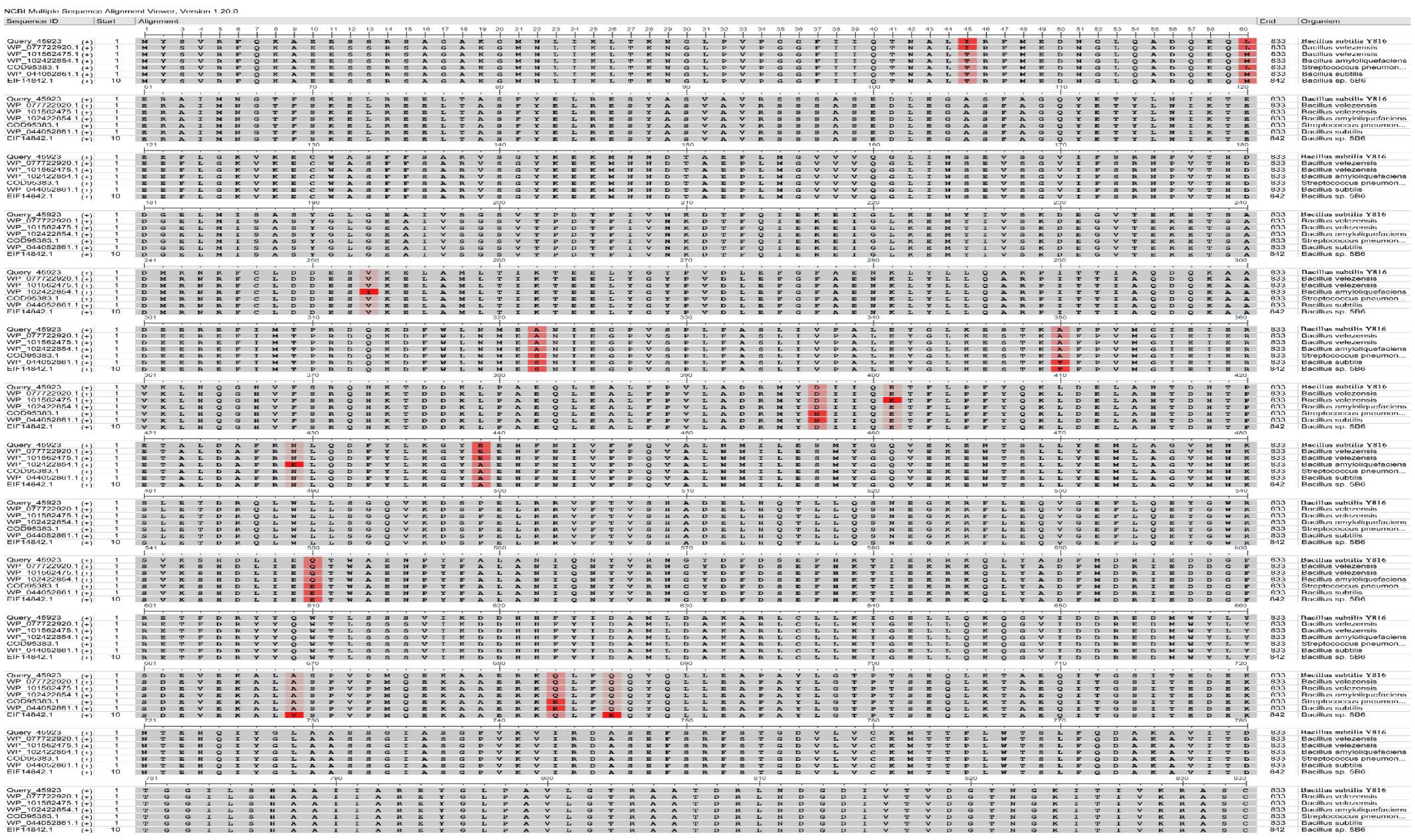


Figure S12. Multiple sequence alignment of phosphotransferase sequences from different strains selected in NCBI.

The selected high sequence identity ( $>98 \%$ ) proteins were from Bacillus velezensis, Bacillus amyloliquefaciens, Streptococcus pneumoniae, Bacillus subtilis, Bacillus species, respectively. ZPH was means by Query_45923. The mismatch residues were highlighted in red with frequency-based difference.
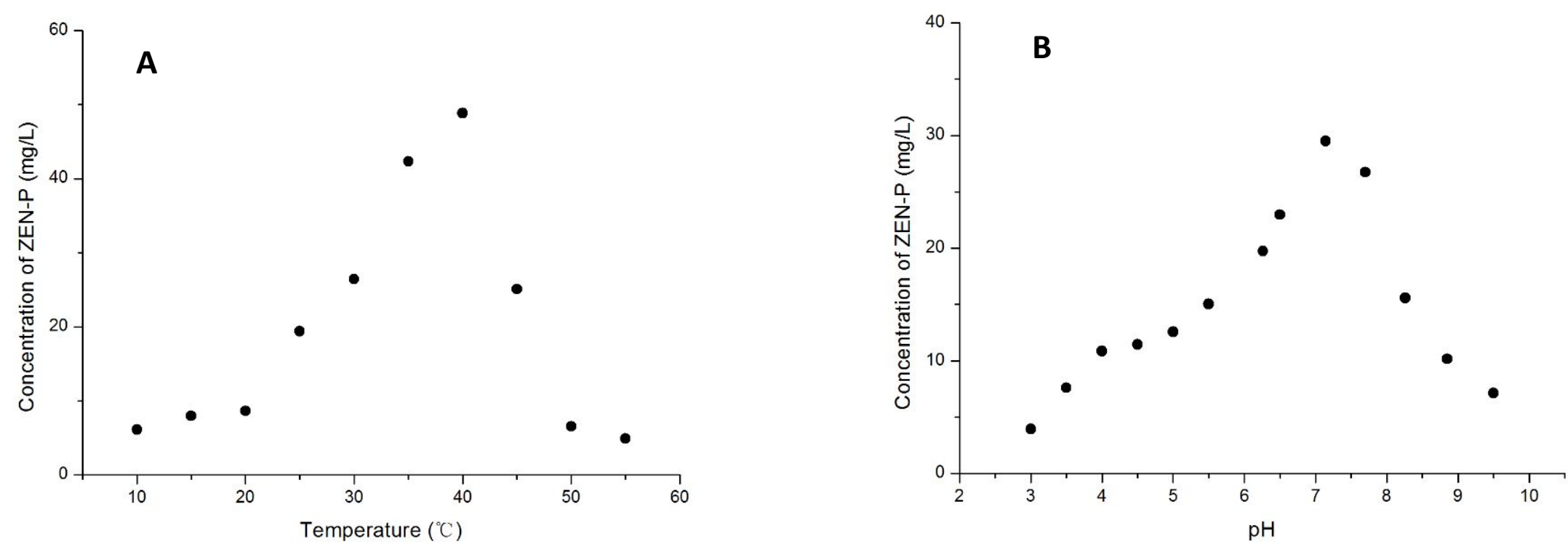

Figure S13. Characteristics of the purified ZPH. A: the optimum temperature of ZPH; B: the optimum pH of ZPH. 